\title{
Design and Development of the ATLAS Central Solenoid Magnet
}

\author{
A. Yamamoto, T. Kondo, Y. Doi, Y. Makida, K. Tanaka, T. Haruyama, H. Yamaoka \\ High Energy Accelerator Research Organization (KEK), Tsukuba, Ibaraki, 305-0801, Japan
}

H. ten Kate, L. Bjorset

European Laboratory for Particle Physics (CERN), CH-1213, Geneva, Switzerland

K. Wada, S. Meguro, The Furukawa Electric Co. Ltd., Nikko, Tochigi, 321-1493, Japan

J.S.H. Ross, and K.D. Smith, Oxford Instruments, Oxford, OX2 0DX, England

\begin{abstract}
The ATLAS central solenoid magnet is being constructed to provide a magnetic field of 2 Tesla in the central tracking part of the ATLAS detector. As a key technology for the solenoid coil as thin as possible, high-strength aluminum stabilized superconductor has been developed with achieving a yield strength of $>100 \mathrm{MPa}$ at $4.2 \mathrm{~K}$. This paper describes the status of the design and development of the solenoid magnet.
\end{abstract}

\section{INTRODUCTION}

The ATLAS central solenoid magnet is being constructed as a collaborative work between KEK and CERN for the ATLAS experiment in the LHC project [1]-[3]. The solenoid is required to provide a axial magnetic field of 2 Tesla in a warm-bore of $2.3 \mathrm{~m}$ in diameter for precise momentum measurement of charged particles produced in $14 \mathrm{TeV}$ proton-proton interactions at LHC. Figure 1 shows a bird'seye view of the ATLAS central solenoid.

Since the coil is placed in front of the liquid-argon (LAr) electromagnetic calorimeter, the solenoid coil must be as thin (and transparent) as possible for the best calorimeter performance with minimum interactions of elementary particles in the coil. The solenoid coil design is featured with high-strength aluminum stabilized super-conductor to make the solenoid coil thinnest while keeping its electrical stability. As a further effort, the solenoid coil is installed in a common

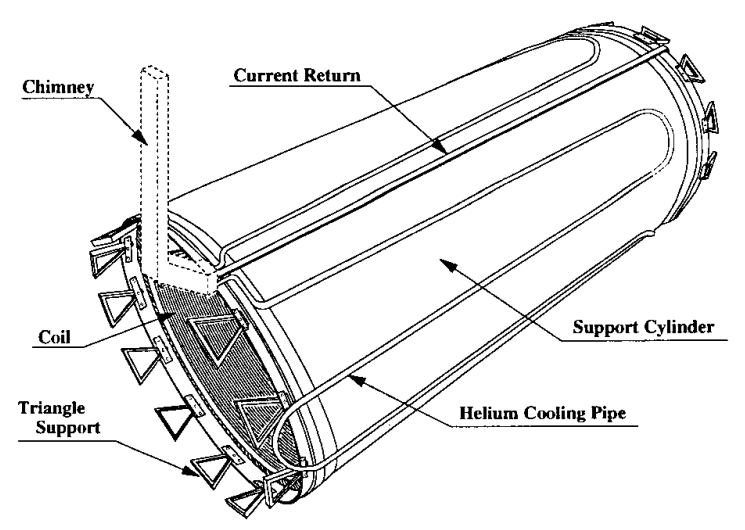

Fig. 1. Schematic view of the ATLAS central solenoid.

Manuscript received on Sept. 14, 1998 vacuum vessel with the LAr calorimeter to minimize the wall material. Since the magnetic return yoke is located enough away, the coil is designed to be an iron-free, air-core solenoid. Fig. 2 shows the cross sectional view of the solenoid coil installed in the LAr calorimeter cryostat. This paper describes progress in design and fabrication of the ATLAS central solenoid magnet.

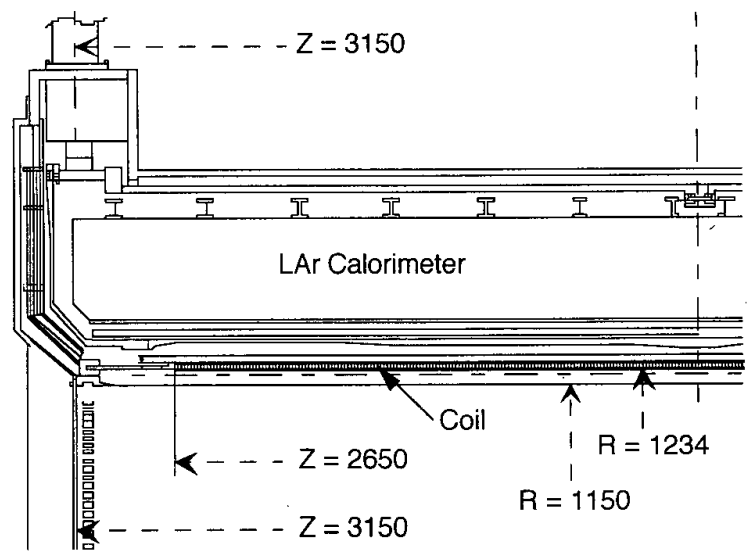

Fig. 2. Cross section of the coil, LAr calorimeter, and the common cryostat.

\section{MAGNET DESIGN}

\section{A. Superconducting Coil}

The ATLAS central solenoid magnet was designed with the following guide-lines [4], [5].

- Single layer coil with edge-wise winding, inside the coil support cylinder

- Mechanically elastic design with a stress intensity level of $70 \mathrm{MPa}$, with keeping a strain $\leq 0.1 \%$,

- Magnetic stored energy fully absorbable in the coil, with keeping a coil temperature $\leq 120 \mathrm{~K}$, in a quench,

- Thin coil with $\leq 0.7$ Xo (radiation thickness) optimized with an $\mathrm{E} / \mathrm{M}$ (stored energy/coil mass) ratio of $7-8 \mathrm{~kJ} / \mathrm{kg}$,

- Indirect cooling by using two-phase helium flow.

Design parameters of the solenoid magnet are summarized in Table I, and the transparency breakdown is given in Table. II. 
TABLE I

DESIGN PARAMETERS OF THE ATLAS CENTRAL SOLENOID

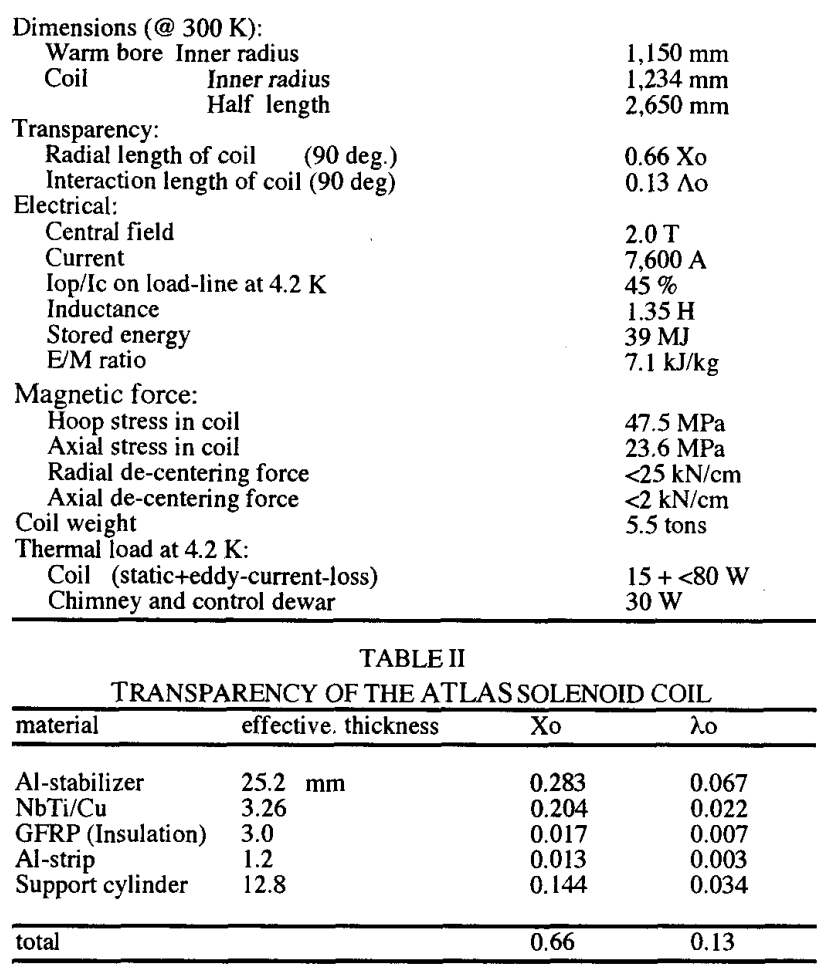

Figure 3 shows the cross section of the coil. The necessary coil thickness, $t$, to support the radial magnetic pressure, may be given by $t=r \cdot p / \sigma_{h}$, where $r$ is the coil radius, $p$ is the magnetic pressure, and $\sigma_{h}$ is the hoop stress in the coil. Since it is proportional to " $r$ " and $\mathrm{B}^{2}$, we may consider a useful parameter to scale the transparency of the superconducting coil in terms of the ratio of the stored energy, E, to the coil cold mass, $M$. This ratio can be simplified by:

$$
\mathrm{E} / \mathrm{M}=\left\{\mathrm{B}^{2} / 2 \mu_{0} \cdot \mathrm{V}_{\mathrm{f}}\right\} /\left\{\mathrm{v}_{\mathrm{c}} \cdot \rho\right\} \approx \sigma_{\mathrm{h}} / 2 \rho,
$$

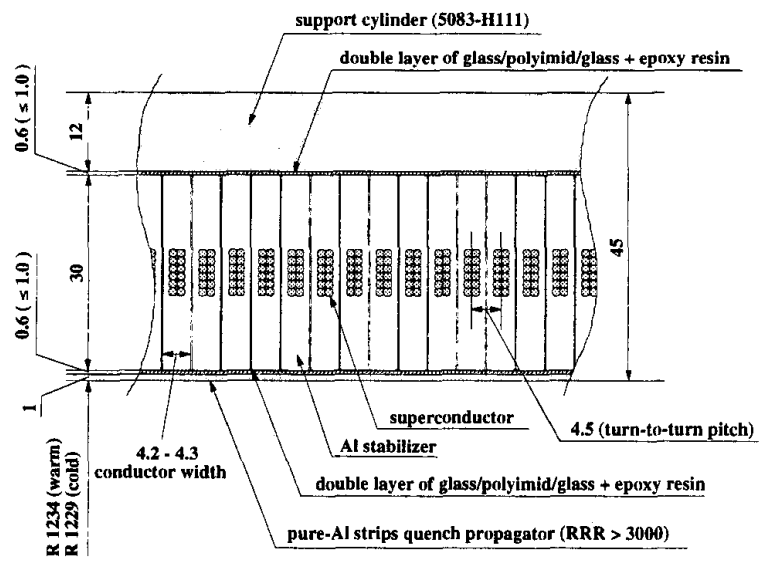

Fig. 3. Cross section of the ATLAS solenoid coil. where $V_{f}\left(=\pi r^{2}\right)$ is the magnetic field volume per length, $\mu_{0}$ is the permeability, $v_{c}(=2 \pi r t)$ is the coil volume per length, and $\rho$ is the effective density of coil material. In case of the aircore solenoid having additional axial stress component corresponding to a half level of the hoop stress, the stress intensity $\left(\sigma_{S I}=\sigma_{h}-\sigma_{z}=1.5 \sigma_{h}\right)$ should be additional constraint to be enough below yield strength $\left(\mathrm{YS}_{0.2}\right)$ in the mechanically elastic design. Figure 4 . shows the $\mathrm{E} / \mathrm{M}$ ratio in various superconducting solenoid magnets for particle detectors in high energy physics. In the ATLAS solenoid, an E/M ratio of $7.1 \mathrm{~kJ} / \mathrm{kg}$ is expected in operation in the ATLAS detector (and $8 \mathrm{~kJ} / \mathrm{kg}$ in the magnet performance test at I$\max =8,400 \mathrm{~A}$ in air). Our previous experience in the SDCprototype solenoid has already demonstrated the E/M ratio of about $10 \mathrm{~kJ} / \mathrm{kg}$ including full energy dump test, and the ATLAS solenoid design should be safe enough [6].

The E/M ratio is also approximately equal to the coil enthalpy and is related to the coil temperature when the stored energy is dissipated entirely and uniformly in the coil after quench as follows:

$$
E / M=\int C_{p} d T \approx H(T),
$$

where $C_{p}$ is the specific heat of the coil (aluminum), $H(T)$ is enthalpy of the coil at the temperature $\mathrm{T}$ after a quench. The $\mathrm{E} / \mathrm{M}$ ratio of $7 \mathrm{~kJ} / \mathrm{kg}$ corresponds to $\mathrm{T}=75 \mathrm{~K}$ and the coil is thermally safe enough, even including some non-uniform temperature distribution in the coil. The uniform energy dump can be realized by using a quench propagator of purealuminum axial strips. This technique was established in the previously developed coils [6,7]. In the ATLAS central solenoid, pure $(99.9998 \%)$ aluminum strips of $10 \mathrm{~cm}$ in width and $1.2 \mathrm{~mm}$ in thickness are glued onto inner surface of the coil [8]. The maximum coil temperature was expected to be less than $120-\mathrm{K}$ by using pure aluminum quench propagator with RRR $>3,000$, as given in Table III.

\section{B. Cooling of the magnet}

The solenoid coil is cooled indirectly by using two-phase helium flow [9]. A helium cooling tube with an inner

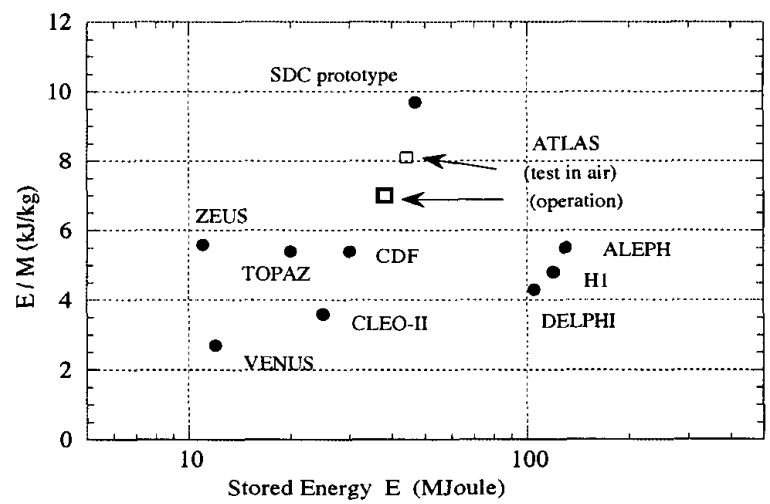

Fig. 4. E/M Ratios in various detector solenoid. 
TABLE III

PEAK COIL TEMPERATURE AFTER QUENCH

\begin{tabular}{lll}
\hline RRR of pure-Al strip & Decay time & T-peak \\
\hline & $30 \mathrm{sec}$ & $134 \mathrm{~K}$ \\
1500 & 24 & 130 \\
2000 & 23 & 120 \\
3000 & 23 & 116 \\
4000 &
\end{tabular}

diameter of $18 \mathrm{~mm}$ is welded to the outer surface of the support cylinder. It has a pair of 6 parallel serpentine paths from the bottom to the top of the support cylinder. A forced flow of two-phase helium is taken in steady state operation. A thermosyphon cooling is prepared for a back-up. Figure 5 shows operational cryogenics characteristics in a temperature-entropy (T-S) diagram in He thermodynamics.

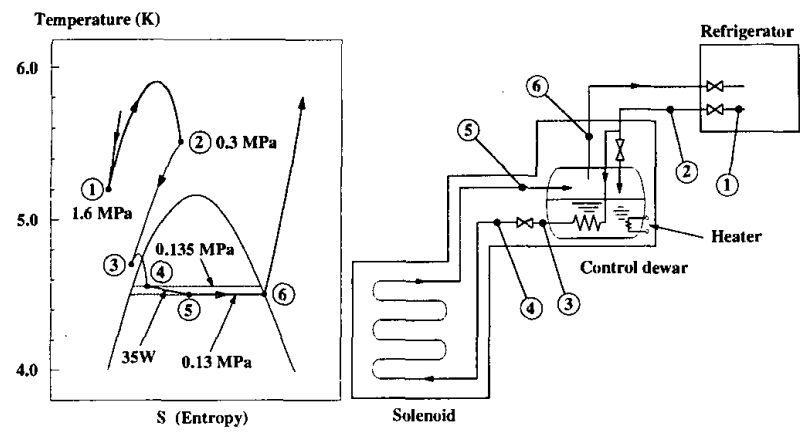

Fig. 5. The cryogenics flow diagram and characteristics.

\section{FABRICATION}

\section{A. Superconductor}

The superconductor consists of Rutherford type $\mathrm{NbTi} / \mathrm{Cu}$ superconducting cable and aluminum stabilizer, as shown in Fig. 6. A half of the superconductor has been fabricated by the Furukawa Electric Co. Ltd., and another half is being fabricated by Hitachi Cable Co. Ltd. The critical current characteristics are shown in Fig. 7. Design parameters and measured performance by Furukawa are given in Table IV.

An extensive R\&D effort has been made to improve the mechanical strength of aluminum stabilized superconductor while keeping adequate residual resistance ratio (RRR) [10]. The following process has been established;

- Micro-alloying: pure(5N)-Al alloyed with additives,

- Mechanical cold work: area reduction.

As a result of the development, the yield strength of 110 $\mathrm{MPa}$ (at RRR = 592) was achieved by using an additive of $\mathrm{Ni}$ (1000-ppm) in combination of a cold work of $21 \%$ [11]. The measured mechanical strength is given in Table $\mathrm{V}$. The stress-strain curve of aluminum stabilizer is shown in Fig. 8, compared with A5083 for the support cylinder. The strength
TABLEIV

CHARACTERISTICS OF Al-STABILIZED SUPERCONDUCTOR

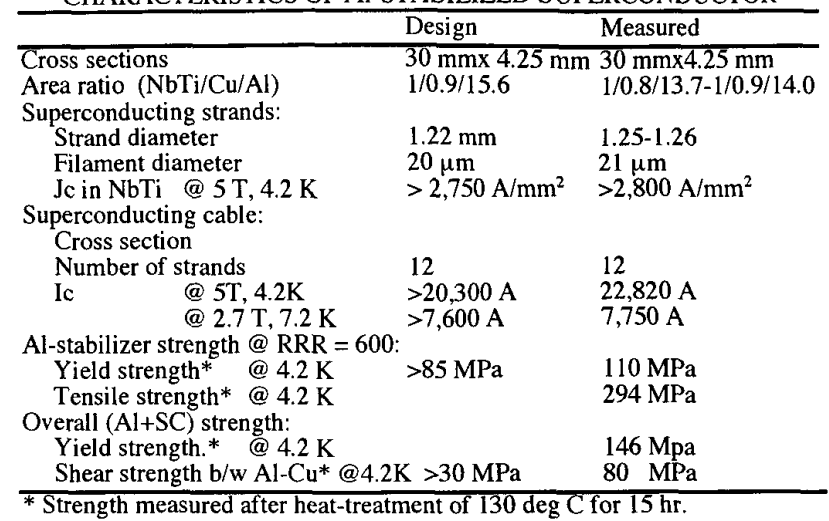

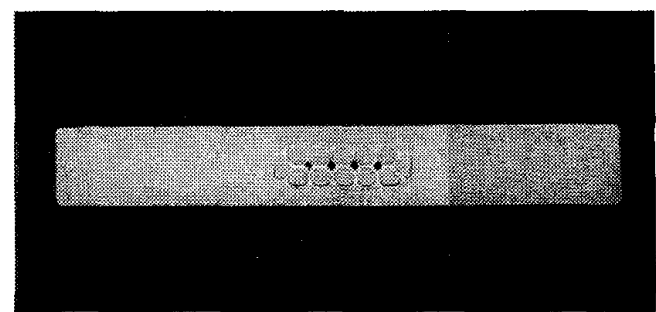

Fig. 6. Aluminum stabilized superconductor cross section.

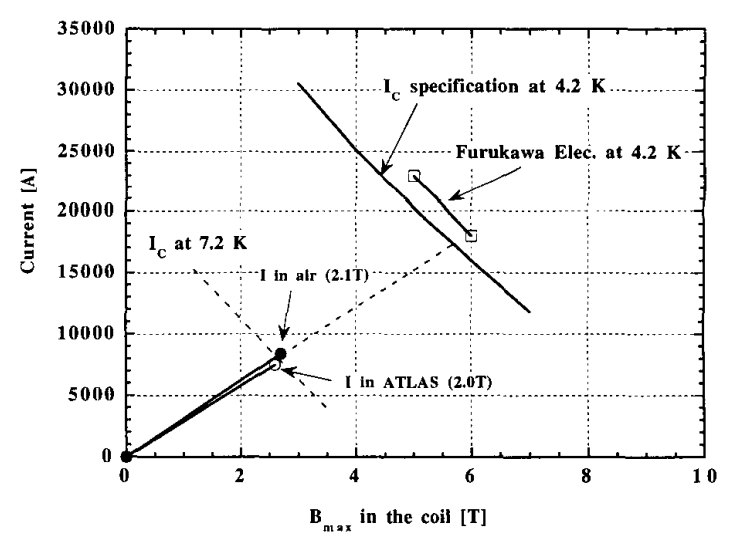

Fig. 7. Critical current characteristics of the superconductor and the solenoid load line characteristics.

TABLE V

MECHANICAL STRENGTH OF AL-STABILIZER AND CONDUCTOR. AFTER CURING AT $130^{\circ} \mathrm{C}$ FOR 15 HOURS.

\begin{tabular}{llll}
\hline & Temp. & $0.2 \%$ Y. S. & T. S. \\
\hline \multirow{2}{*}{ Aluminum Stabilizer: } & $300 \mathrm{~K}$ & $81 \mathrm{MPa}$ & $86 \mathrm{MPa}$ \\
& 77 & 99 & 157 \\
& $4.2 \mathrm{~K}$ & 110 & 294 \\
& & & \\
Over-all (AL+NbTi/Cu) & $300 \mathrm{~K}$ & 128 & 184 \\
& $77 \mathrm{~K}$ & 131 & --- \\
& $4.2 \mathrm{~K}$ & 147 & -- \\
\hline
\end{tabular}




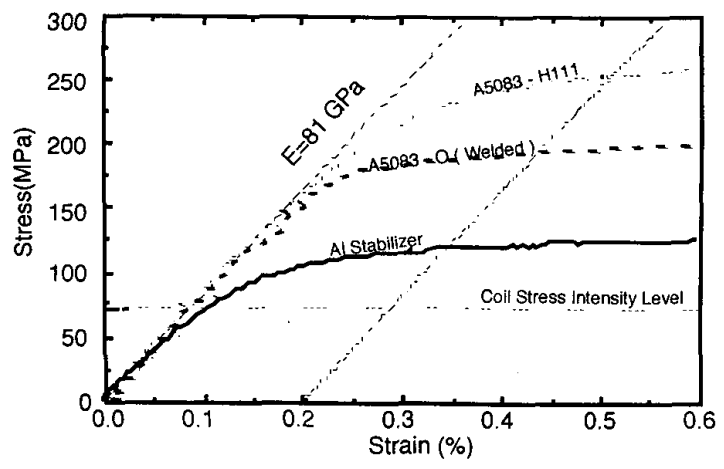

Fig. 8. Stress-strain curve of Al stabilizer compared with A-5083.

was obtained in the measurement after a heat treatment at 130 ${ }^{\circ} \mathrm{C}$ for 15 hours to take into account of coil curing after the coil winding. Fig. 9 shows the yield strength of aluminum stabilizer at $4.2 \mathrm{~K}$ as a function of RRR. Basically the additives contribute to improve the strength with less contribution to increasing the electrical resistance. The mechanical cold work has a major effect to improve the mechanical strength. The micro-alloying may contribute to keep its mechanical stability during and after the coil curing process at $130^{\circ} \mathrm{C}$. Further details are still to be investigated.

\section{B. Solenoid Coil}

The solenoid coil is wound inside the coil support cylinder, which has been fabricated by Oxford Instruments, UK, in cooperation with Romabau, Switzerland. Figure 10 shows the picture of the support cylinder completed. Cooling tubes are welded on the outer surface, and two end-rings are attached by using bolts to interface triangle-shape coil supports from the vacuum vessel. Polyimide film, Upilex, has been chosen for electrical insulation The film is preprocessed with glasscloth and epoxy-resin (GUG) for the best electrical and bonding performance [12], [13]. The single layer coil is to be wound directly inside the coil support cylinder with turn-toturn insulation by using a laminated tape of Upilex and epoxy-resin. Inside the coil, the pure-Al strip quench propagators will be glued in axial direction after ground plane

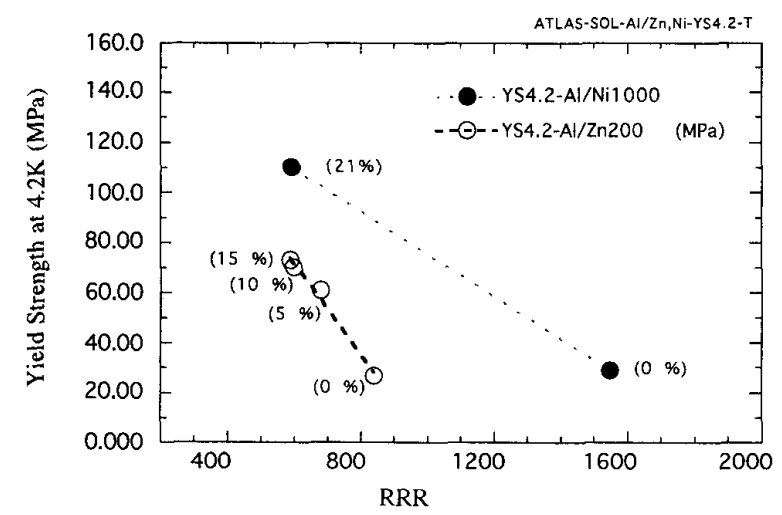

Fig. 9. Yield strength v.s. RRR of high-strength $\mathrm{Al}$ stabilizer. insulation work inside. The coil winding is being prepared by Toshiba, Japan. The coil is to be supported by 12 triangleshaped coil supports made of GFRP at each axial end. The supports have been fabricated by Arisawa Mfg. Company. As a test result, each support can sustain more than $50 \mathrm{kN}$ with keeping a safely factor of $>4$ against electromagnetic de-centering force [14].

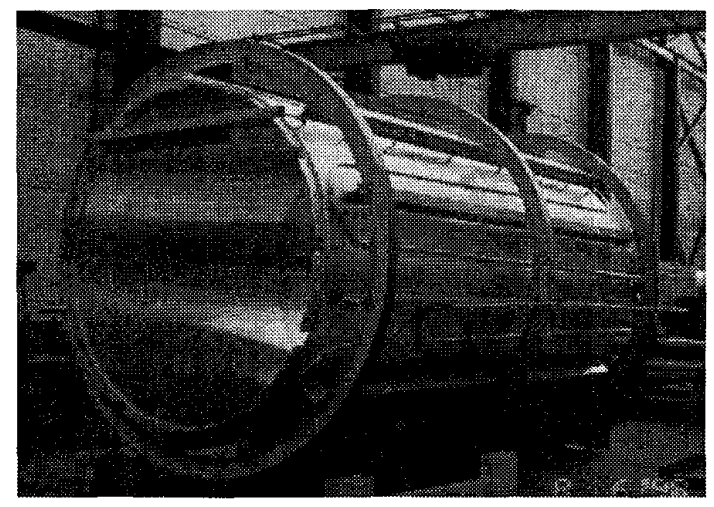

Fig. 10. Picture of the coil support cylinder

\section{SUMMARY}

The ATLAS central solenoid magnet was designed to provide a magnetic field of $2 \mathrm{~T}$ in a warm volume of $2.3 \mathrm{~m}$ in diameter and $6.3 \mathrm{~m}$ in length with an E/M ratio of $7 \mathrm{~kJ} / \mathrm{kg}$. A coil transparency of 0.66 radiation length is expected in the design featured with high-strength aluminum stabilized superconductor at a yield strength of $>100 \mathrm{MPa}$ at $4.2 \mathrm{~K}$ with $R R R=600$. The coil fabrication is in progress and is to be completed in 2000 .

\section{ACKNOWLEDGMENT}

The authors would like to thank the ATLAS collaboration for continuous support and encouragement to proceed this work. They would like to thank Romabau $A G$, Switzerland for the cooperative work with Oxford Instruments Co. Ltd. The KEK-team would like to thank Dr. J. Sondericker and his team at BNL for their cooperative work in interfaces with LAr calorimeter. Finally, the authors would thank KEK and CERN for continuous support and encouragement in this work.

\section{REFERENCES}

[1] ATLAS Technical proposal, CERN/LHCC/94-43, LHCC/P2 (1994).

[2] H. ten Kate et al, "ATLAS magnet system, Technical Design Report" CERN/LHCC/97-20, ATLAS TDR 9, 1997.

[3] A. Yamamoto et al, ATLAS Central Solenoid Technical Design Report, CERN/LHCC/97-2, ATLAS-TDR-9, 1997.

[4] Y. Makida et al, IEEE Trans. Appl. Supercond., No. 7 1997, p. 638.

[5] K. Tanaka et al, Proc. ICEC-16, Kita-kyushu, Japan, 1996, p. 1 19-122.

[6] A. Yamamoto et al, IEEE Trans. Appl. Super. Vol. 5, 1996, P.849.

[7] Y. Makida et al., Adv. Cryog. Eng. 37, 1991, p. 401-407.

[8] A. Takahashi et al, developed by Sumitomo-Chemical Co. Ltd.

[9] T. Haruyama et al., Cryogenics 36,1996, p. 465-469.

[10] I. Inoue et al, Supercollider, No. 4, 1992, p.943. K. Wada, Furukawa Electric. Co., personal communication, 1998

[11] F.R. Fickett, Cryogenics, 1971, p. 349-367.

[12] T. Ueki et al, to be published in Adv. in Cryog. Eng. Vol. 44.

[13] E. Evans et al, to be published in Adv. in Cryog. Eng., Vol. 44.

[14] H. Yamaoka, et al, Proc. MT15, Beijing, 1997, p. 204-207. 